\title{
Non-Steroidal Anti-Inflammatory Drug Ingestion as a Risk Factor to Anaphylaxis During Immunotherapy: a Case Series
}

\section{David C Fahmy ${ }^{1}$ and Jason K Lee ${ }^{2 *}$}

${ }^{1}$ UHN Toronto General Hospital, 190 Elizabeth St. Toronto, ON M5G 2C4, Canada

${ }^{2}$ FRCPC, St. Michael's Hospital Clinical Immunology and Allergy Clinic, 4 Cardinal Carter Specialty Clinics, 30 Bond Street, Toronto, Ontario M5B 1W8, Canada

\begin{abstract}
Background: Subcutaneous allergen immunotherapy (SCIT) is a common treatment for seasonal and/or perennial rhinitis, conjunctivitis, or asthma. Unfortunately, adverse events may occur during treatment with allergen immunotherapy (AIT), including systemic reactions that may range in severity from cutaneous manifestations to anaphylaxis.

Objectives: Although the effect of acetasalicylic acid (ASA) and other non-steroidal anti-inflammatory drugs (NSAIDs) on mast cells and as cofactors of anaphylaxis have been well-described, their role in the setting of AIT has not. The current practice parameters do not address NSAIDs as a potential risk factor for anaphylaxis with AIT. This article provides a series of cases that offer evidence that these medications should also be used with caution when administering AIT.

Results: We describe six cases of patients with various environmental allergies that had been undergoing AIT and experienced anaphylaxis. On history, each of these patients had ingested ASA or NSAIDs within 24 hours of the injection. Four out of the six described patients elected to continue AIT and remain on maintenance doses without incident. These patients made no additional changes with the exception of avoiding NSAIDs 24 hours prior to injection.

Conclusions: These cases may bring to attention the role of ASA and other NSAIDs in acting as a co-factor for anaphylaxis in the setting of SCIT. Physicians providing immunotherapy may wish to ensure that their discussion of the risks and benefits of the treatment include information that ASA and NSAID use prior to receiving therapy may increase the risk of a systemic reaction. Patients may wish to use a safer alternative if such exists.
\end{abstract}

Keywords: Immunotherapy; ASA: Acetylsalicylic acid; NSAID: Non-Steroidal Anti-Inflammatory Drug; Ibuprofen; Anaphylaxis; Systemic reaction; Adverse event

\section{Background}

Subcutaneous allergen Immunotherapy (SCIT) is a common treatment for seasonal and/or perennial rhinitis, conjunctivitis, or asthma.

Unfortunately, adverse events may occur during treatment with Allergen Immunotherapy (AIT), including systemic reactions that may range in severity from cutaneous manifestations to anaphylaxis [1].

To better understand the epidemiology of systemic reactions, the American Academy of Allergy Asthma and Immunology (AAAAI) and the American College of Allergy Asthma and Immunology (ACAAI) examines this periodically. The AAAAI initiative, using self-reported data, captures the activity of over 1900 North American prescribers [2]. A recent study showed that approximately 0.1 percent of injections elicited a systemic reaction of varying severity [3]. Anaphylactic reactions (grade 3 on their scale or higher) represented $3-4 \%$ of all manifestations, with no reported deaths [3]. Previous retrospective studies estimate, a fatal reaction occurring once every 2.5 million injections from 1990 to 2001 [4].

Severe or even fatal reactions may be associated with several risk factors [5]. The most common risk factors include uncontrolled asthma, a prior history of systemic reactions to AIT, administration of injections during peak pollen season, or delay in prompt administration of epinephrine [4].

Currently, two medications are listed in the guidelines as possible contraindications to AIT [5]. The first class of medications is beta- blockers, which it states may cause more serious and treatment-resistant anaphylaxis [5]. Although not an absolute contraindication, alternative treatments, if possible, are recommended if allergen AIT is to be administered in a patient with previous anaphylaxis [5]. The second class of medications is the angiotensin converting enzyme (ACE)inhibitors. ACE-inhibitors have been associated with greater risk of severe reactions, particularly with venom AIT [5]. Mechanistically, ACE-inhibitors prevent the breakdown of vasoactive kinins during an anaphylactic reaction [5]. Evidence of this was shown in two patients who had anaphylaxis to venom AIT while on ACE inhibitor treatment, but not when the drug was withheld [5].

Although the effects of Acetasalicylic Acid (ASA) and other NonSteroidal Anti-Inflammatory Drugs (NSAIDs) on mast cells and as cofactors of anaphylaxis have been well-described, their role in the setting of AIT has not. The current practice parameters do not address NSAIDs as a potential risk factor for anaphylaxis with AIT.

*Corresponding author: Jason K Lee, FRCPC, St. Michael's Hospital Clinica Immunology and Allergy Clinic, 4 Cardinal Carter Specialty Clinics, 30 Bond Street, Toronto, Ontario M5B 1W8, Canada, Tel: 4168646060; Fax: 8645784; E-mail: jasonk.lee@utoronto.ca

Received June 12, 2013; Accepted July 18, 2014; Published July 29, 2014

Citation: Fahmy DC, Lee JK (2014) Non-Steroidal Anti-Inflammatory Drug Ingestion as a Risk Factor to Anaphylaxis During Immunotherapy: a Case Series. J Allergy Ther 5: 181. doi:10.4172/2155-6121.1000181

Copyright: @ 2014 Fahmy DC, et al. This is an open-access article distributed under the terms of the Creative Commons Attribution License, which permits unrestricted use, distribution, and reproduction in any medium, provided the original author and source are credited. 


\section{ASA, the Mast Cell, and Allergy Provocation}

ASA and other NSAIDs have a well-documented role in causing hypersensitivity reactions, collectively known as Aspirin intolerance, which can include urticaria, asthma, and anaphylaxis [6].

ASA has been shown to induce expression of heat shock proteins, particularly HSP70 [7]. Mast cells have been shown to produce and release heat-shock proteins into the extracellular environment, which works through an autocrine fashion to activate mast cells to produce cytokines [8]. The investigators were able to show that the heat-shock proteins were able to induce several cytokines including TNF-a and IL-6 through the mast cell's TLR4 receptor pathway [8].

Another important feature of aspirin intolerance appears to be the overproduction of cysteinyl leukotrienes (cys-LTs) [6]. Based on the observation that cys-LTs were in higher concentrations in the bronchoalveolar lavage of patients with aspirin intolerance, a study was designed to examine the effects of ASA on IgE-mediated LT secretion [6]. Togo et al. created a mast cell with an IgE antibody to an experimental antigen. They exposed the cell to ASA, followed by the antigen. At concentrations equivalent to therapeutic doses of ASA, LT secretion was increased in IgE-mediated reactions, while ASA alone had no effect on LT release [6]. This was shown to be a separate mechanism from prostaglandin, as the investigators used an aspirin derivative that was missing the essential acetyl component. Instead, they showed that the effect was through a dihydropyridine-receptor mediated calcium channel [6].

A recent review of anaphylaxis lists ASA and NSAIDs as extrinsic risk factors to the development of anaphylaxis [9].

Clinically, this "priming" effect of ASA has been described in several settings, particularly in Food-Derived Exercise-Induced Anaphylaxis (FDEIA) [10]. A case series of three patients with specific IgE antibodies to food (wheat or shrimp), showed they were able to elicit a systemic allergic response when ingesting ASA first followed by the food, but no reaction when given either ASA or the food alone [10]. In a small clinical trial, five of seven patients had a systemic allergic response to an established food allergen by skin prick test (SPT) or specific IgE only when pretreated with oral aspirin, but not with the food alone [11].

To this point, the role of ASA in inducing reactions to aeroallergens and specifically to AIT has not been reported. We provide a series of cases that provide evidence for the role of ASA as a risk factor for inducing anaphylaxis in patients receiving SCIT.

\section{Index Cases}

\section{Patient A}

Patient A was a 29 year old male with a history of asthma, eczema, and allergic rhinitis. He was SPT positive to rye grass, Dermatophagoides pteronyssinus (dust p), Dermatophagoides farinae (dust f), grass mix, alder red, cat pelt, cat hair, birch (ALK skin prick test extracts) and was started on allergen immunotherapy (AIT) by Hollister Stier for dust mite mixture (1000 AU/ml each), cat (2000 BAU/ml), grasses (5000 $\mathrm{PNU} / \mathrm{ml}$ ), and trees (tree mix $5000 \mathrm{PNU} / \mathrm{ml}$ ). He was otherwise healthy and his only medication was cetirizine, a non-sedating antihistamine.

This patient received an AIT injection midway through the final vial in a three-vial set, which he had been tolerating to that point with minimal skin symptoms. Within one hour, the patient began to develop diffuse urticaria and shortness of breath. The patient was administered
$0.3 \mathrm{mg}$ epinephrine IM using an epinephrine auto-injector. The patient's symptoms resolved and he was discharged home after several hours of observation with an epinephrine auto-injector and instructions to go to the emergency department if symptoms recurred. On history, the patient reported using ibuprofen the morning of his injection for mild MSK pain. He took no other medications, otherwise.

The patient has since elected not to continue AIT due to the severity of his symptoms.

\section{Patient B}

Patient B was a 23 year old male with a history of allergic rhinitis. He was SPT positive to rye grass, dust $\mathrm{p}$, dust $\mathrm{f}$, alder red, cat pelt, cat hair, dog, birch, white ash (ALK skin prick test extracts) and was started on ALK AIT for dust (1000 AU/ml), cat (1000 BAU/ml), grass $(5000$ $\mathrm{PNU}$ ), and trees (tree mix $5000 \mathrm{PNU} / \mathrm{ml}$ ). He was otherwise healthy and his only medication was Ciclesonide nasal spray.

The patient received an AIT injection to a buildup dose in vial \#2 of a 3 vial set, which he had been tolerating to that point with minimal skin symptoms. Within five minutes of the injection, the patient began to develop presyncope and nausea followed by vomiting. Emergency medical services (EMS) were called and the patient was administered $0.3 \mathrm{mg}$ epinephrine IM using an epinephrine auto-injector with $100 \%$ oxygen via high flow face mask. While awaiting EMS, symptoms persisted and he was given a second dose of $0.3 \mathrm{mg}$ epinephrine five minutes later. The attending physician accompanied the patient to the ER as the patient became incoherent and hypotensive with a blood pressure of 90/60. During the transfer, intubation was considered when the GCS score was 10 . The patient was given a third dose of epinephrine at $0.5 \mathrm{mg}$ IM upon arrival to the ER. The patient's symptoms resolved after a day and he was admitted under internal medicine and discharged home the next day. In follow-up, the patient reported that he had been using ibuprofen daily for the week prior to his injection due to a skiing accident and ongoing headaches. He had been taking no other medications, except for the nasal steroid.

This patient highly desired to continue with AIT and asked the attending allergist on over three separate occasions to restart, however given the severity of his reaction he was not continued on AIT.

\section{Patient C}

Patient $C$ was a 20 year old male with a history of allergic rhinitis. He was SPT positive to rye grass, dust $\mathrm{p}$, dust $\mathrm{f}$, alder red, cat pelt, cat hair, birch, tree mix and ragweed and was started on pre-seasonal AIT $\left(\right.$ Center- $\left.\mathrm{AL}^{\circledR}\right)$ for trees and grasses in January of 2012. He was otherwise healthy and took no medications.

On February 1, 2012, he received an AIT injection midway in the buildup, which he had been tolerating to that point with minimal skin symptoms. Within two hours, the patient began to develop diffuse urticaria and pruritus, but remained hemodynamically stable. He felt some subjective throat discomfort but this resolved by the time he returned to be assessed 2 hours post AIT. The patient was administered $0.3 \mathrm{mg}$ epinephrine IM using an epinephrine auto-injector and cetirizine $20 \mathrm{mg}$ orally. The patient was taken to the emergency department and was discharged home after 2 hours of observation with an epinephrine auto-injector and instructions to return to the emergency department if symptoms recurred. On history, the patient reported using ibuprofen prior to his injection for a headache. He took no other medications, otherwise.

After discussion of risks and benefits, AIT was resumed building 
up again from a lower concentration, which he tolerated, and moved to higher concentrations the week after, which he tolerated as well and completed.

\section{Patient D}

Patient D was a 42 year old female with a history of allergic rhinitis. She was SPT positive to dust $\mathrm{p}$, dust $\mathrm{f}$, cat pelt, cat hair, grass mix, rye grass, birch, alder red, tree mix, weed mix, goldenrod, cockroach, dandelion, rabbit, mouse and feather. On history, she was most symptomatic to cat and began AIT only for cat $(1000 \mathrm{BAU} / \mathrm{ml})$ (Hollister-Stier). The patient's other medications included Mirena 52 $\mathrm{mg}$ oral and Ativan $0.5 \mathrm{mg}$ orally as needed for sleep.

The patient received an AIT injection midway through the final vial in a 4 vial set, which she had been tolerating to that point with minimal skin symptoms. Within two hours, the patient began to develop diffuse urticaria and eyelid angioedema, but remained hemodynamically stable. She felt subjective chest tightness. She took loratadine $10 \mathrm{mg}$ and went to the emergency department where she received another oral antihistamine and was observed until her symptoms dissipated. She was provided an epinephrine auto-injector and instructions to return to the emergency department if symptoms recurred. On history, the patient reported using ibuprofen the night before and morning of her injection for knee pain. She took her other medications as prescribed.

After discussion of risks and benefits, AIT was resumed starting from 3 levels below the dose at which she had the urticaria and angioedema reaction. She has avoided NSAIDs prior to the injections but has taken her other medications regularly and continued to tolerate the injections with no significant reactions.

\section{Patient E}

Patient E was a 30 year old female with a history of allergic rhinoconjunctivitis. She was SPT positive to dust $p$, dust $f$, and cat and was started on AIT for cat. She was otherwise healthy and took no medications.

The patient received an AIT injection near the end of vial 3 in a 3 vial set, which she had been tolerating previously without significant systemic symptoms. Within 15 minutes, the patient began to develop diffuse urticaria, facial swelling, and shortness of breath. The patient was administered $0.3 \mathrm{mg}$ epinephrine IM using an epinephrine autoinjector and given $100 \%$ supplemental oxygen by facemask. She maintained her blood pressure. Due to worsening urticaria, the patient was given a further $0.15 \mathrm{mg}$ of epinephrine in the left thigh. She was given cetirizine $20 \mathrm{mg}$ and observed. Her symptoms resolved and she was monitored for 2 hours. The patient was given an epinephrine autoinjector and discharged home with instructions to seek medical attention if her symptoms returned. On questioning, the patient reported taking two extra strength ibuprofens for some neck pain approximately two hours prior to AIT.

After discussion of risks and benefits, AIT for cat was successfully resumed with NSAID avoidance on days of AIT. To date she continues on full maintenance dose.

\section{Patient F}

Patient F was a 27 year old female with a history of asthma and allergic rhinoconjunctivitis. Her SPT was positive to rye grass, dust $\mathrm{p}$, dust f, cat pelt, cat hair, grass mix, ragweed, rabbit, and mouse. She was started on AIT for dust mite and cat. She was otherwise healthy and took no medications.

The patient received a routine maintenance dose and began to develop diffuse urticaria and facial swelling. She complained of a sore throat at the time of the reaction. She was given epinephrine $0.3 \mathrm{mg}$, which alleviated the aforementioned symptoms. She was monitored for two hours and the symptoms resolved. She was provided with an epinephrine auto-injector and provided with instructions to return to the emergency department if symptoms returned.

Her only cofactor for anaphylaxis was ASA, which she had taken for a headache earlier that morning. After discussion of risks benefits, and alternatives of AIT, the patient successfully resumed AIT with a rebuild up starting three doses back and continues to do well on AIT avoiding NSAIDs and ASA on the days of AIT (Table 1).

\section{Discussion}

The above cases illustrate the role of aspirin and other NSAIDs (mainly ibuprofen) in acting as a co-factor for anaphylaxis in the setting of SCIT. Subcutaneous allergen immunotherapy is a safe and effective treatment for allergic rhinitis, conjunctivitis, and asthma. Systemic reactions are rare (as mentioned earlier, approximately $0.1 \%$ ), but can be minimized through several factors including avoiding certain medications including beta-blockers and ACE inhibitors. Based on the understanding of the mechanisms, as well as the aforementioned cases, ASA and other NSAIDs are probable contributors to adverse events.

Among the patients who reacted, symptoms appeared within 5 minutes to two hours post-injection and urticaria was the commonest symptoms. All but one patient was administered epinephrine in clinic and all recovered fully after a few hours without significant morbidity. In all cases, ASA or NSAIDs were used within 24 hours was a common element. Four out of six patients have safely continued to receive AIT

\begin{tabular}{|c|c|c|c|c|c|c|}
\hline Patient & AIT & NSAID & Time of NSAID & Timing and Nature of Reaction & Management & $\begin{array}{l}\text { Continued } \\
\text { Treatment? }\end{array}$ \\
\hline A & dust mite, cat, grass, tree & ibuprofen & Morning of injection & $\begin{array}{l}1 \text { hour post-injection } \\
\text { Diffuse urticaria, shortness of breath }\end{array}$ & Epinephrine $0.3 \mathrm{mg} \mathrm{IM}$ & No \\
\hline B & dust mite, cat, grass, tree & ibuprofen & $\begin{array}{l}\text { Dailyx } 1 \text { week prior to } \\
\text { injection }\end{array}$ & $\begin{array}{l}5 \text { minutes post-injection } \\
\text { Presyncope, nausea, vomiting, hypotension }\end{array}$ & $\begin{array}{l}\text { Epinephrine } 0.3 \mathrm{mg} \mathrm{IM} \times 2 \\
\text { Epinephrine } 0.5 \mathrm{mg} \mathrm{IM} \times 1\end{array}$ & No \\
\hline C & $\begin{array}{l}\text { Preseasonal trees and } \\
\text { grasses }\end{array}$ & ibuprofen & Morning of injection & $\begin{array}{l}\text { Two hours post-injection } \\
\text { Diffuse urticaria, throat discomfort }\end{array}$ & $\begin{array}{l}\text { Epinephrine } 0.3 \mathrm{mg} \mathrm{IM} \\
\text { Cetirizine } 20 \mathrm{mg} \mathrm{PO}\end{array}$ & Yes \\
\hline D & cat & ibuprofen & $\begin{array}{l}\text { Night prior and } \\
\text { morning of injection }\end{array}$ & $\begin{array}{l}\text { Two hours post-injection } \\
\text { Diffuse urticaria, angioedema, chest tightness }\end{array}$ & Loratadine $10 \mathrm{mg} \mathrm{PO}$ & Yes \\
\hline E & cat & ibuprofen & $\begin{array}{l}\text { Two hours prior to } \\
\text { injection }\end{array}$ & $\begin{array}{l}15 \text { minutes post-injection Diffuse urticaria, } \\
\text { angioedema, shortness of breath }\end{array}$ & $\begin{array}{c}\text { Epinephrine } 0.3 \mathrm{mg} \mathrm{IM} \\
\text { Epinephrine } 0.15 \mathrm{mg} \mathrm{IM} \\
\text { Cetirizine } 20 \mathrm{mg} \mathrm{PO}\end{array}$ & Yes \\
\hline $\mathbf{F}$ & dust mite, cat & ASA & Morning of injection & $\begin{array}{l}15 \text { minutes post-injection } \\
\text { Diffuse urticaria, shortness of breath }\end{array}$ & Epinephrine $0.3 \mathrm{mg} \mathrm{IM}$ & Yes \\
\hline
\end{tabular}

Table 1: Summary of Anaphylaxis Cases. 
without incident and are now on maintenance dose. These patients had no medication changes with the exception of avoiding NSAIDs 24 hours prior to injection. One potential criticism of our study is that NSAIDs and aspirin are commonly used medications and that it may be coincidental that this particular clinic has noticed this as a common co-factor to these reactions. It should be noted however that no other confounding variables on careful history taking were noted.

Randomized control trials to definitively determine the role of ASA and NSAIDs as co-factors of anaphylaxis would be difficult to perform due to the risk of harm. However, the clinical practice guidelines, particularly those for ACE inhibitors were based on a similar body of evidence, including in vitro observations of the mechanism of action and case reports that supported it [5].

At TorontoAllergists.com ${ }^{\circledR}$, the clinic where these reactions occurred, there are two part time allergists and two full time allergists who in total administer approximately 3600 AIT injections annually. The usual post-injection observation period at the office is for 30 minutes. The above cases represent 6 of 8 total cases with a reaction rate calculated at $0.22 \%$, which approximates the reported rate of $0.2 \%$ [3]. Since this observation was noticed, the physicians at TorontoAllergists. com $^{\circledR}$ have proactively asked all AIT patients to refrain from using NSAIDs in the subsequent 12 months from these reactions and it is striking that no further reactions have occurred. Again this may be coincidental and by chance alone. As such, we would welcome further studies on this possibility, particularly one with a larger cohort and an appropriate comparison group.

Although evidence in a controlled setting, with prospective and more robust data, would be welcome, the current body of evidence is likely sufficient to at least allow for consideration of additional caution in administering ASA and NSAIDs during AIT-particularly around the time of injection. Physicians providing AIT should ensure that their discussion of the risks and benefits of the treatment include information that ASA and NSAID use prior to receiving therapy may increase the risk of a systemic reaction.

\section{Author's Contributions}

1. Dr. David Fahmy-main author of the manuscript. Gathered information from charts, performed chart review, researched background information, and wrote manuscript
2. Dr. Jason Lee-Research supervisor, identified index cases, provided revisions and editing of the manuscript; provided final approval for publication

\section{References}

1. Cox L, Larenas-Linnemann D, Lockey RF, Passalacqua G (2010) Speaking the same language: The World Allergy Organization Subcutaneous Immunotherapy Systemic Reaction Grading System. The Journal of allergy and clinical immunology 125: 569-574

2. Epstein TG, Liss GM, Murphy-Berendts K, Bernstein DI (2013) AAAAl and ACAAI surveillance study of subcutaneous immunotherapy, Year 3: what practices modify the risk of systemic reactions? Annals of allergy, asthma and immunology: official publication of the American College of Allergy, Asthma, andlmmunology 110: 274-278

3. Bernstein DI, Epstein T, Murphy-Berendts K, Liss GM (2010) Surveillance of systemic reactions to subcutaneous immunotherapy injections: year 1 outcomes of the ACAAI and AAAAl collaborative study. Annals of allergy, asthma and immunology: official publication of the American College of Allergy, Asthma and Immunology 104: 530-535

4. Bernstein DI, Wanner M, Borish L, Liss GM; Immunotherapy Committee American Academy of Allergy, et al. (2004) Twelve-year survey of fatal reactions to allergen injections and skin testing: 1990-2001. J Allergy Clin Immunol 113: 1129-1136.

5. Cox L, Nelson H, Lockey R, Calabria C, Chacko T, et al. (2011) Allergen immunotherapy: a practice parameter third update. J Allergy Clin Immunol 127: S1-55.

6. Togo K, Suzuki Y, Yoshimaru T (2009) Aspirin and salicylates modulate lgE-mediated leukotriene secretion in mast cells through a di-hydropyridine receptor-mediated $\mathrm{Ca}^{2+}$ influx. Clinical Immunology 131: 145-156

7. Mortaz E, Redegeld FA, Nijkamp FP, Wong HR, Engels F (2006) Acetylsalicylic acid-induced release of HSP70 from mast cells results in cell activation through TLR pathway. Exp Hematol 34: 8-18.

8. Mortaz E, Engels F, Nijkamp FP, Redegeld FA (2009) New insights on the possible role of mast cells in aspirin-induced asthma. Curr Mol Pharmacol 2 : 182-189.

9. Lee JK, Vadas P (2011) Anaphylaxis: mechanisms and management. Clin Exp Allergy 41: 923-938.

10. Harada S, Horikawa T, Ashida M, Kamo T, Nishioka E, et al. (2001) Aspirin enhances the induction of type I allergic symptoms when combined with food and exercise in patients with food-dependent exercise-induced anaphylaxis. $\mathrm{Br}$ J Dermatol 145: 336-339.

11. Aihara M, Miyazawa M, Osuna H, Tsubaki K, Ikebe T, et al. (2002) Fooddependent exercise-induced anaphylaxis: influence of concurrent aspirin administration on skin testing and provocation. $\mathrm{Br} J$ Dermatol 146: 466-472. 